\title{
Multicolorimetric ELISA biosensors on a paper/polymer hybrid analytical device for visual point-of-care detection of infection diseases
}

\author{
Lei $\mathrm{Ma}^{1} \cdot$ Yousef Abugalyon ${ }^{1} \cdot$ XiuJun $\mathrm{Li}^{1,2}$ (D) \\ Received: 4 March 2021 /Revised: 13 April 2021 / Accepted: 16 April 2021 / Published online: 26 April 2021 \\ (C) Springer-Verlag GmbH Germany, part of Springer Nature 2021
}

\begin{abstract}
Enzyme-linked immunosorbent assay (ELISA) is widely used for the detection of disease biomarkers. However, it utilizes timeconsuming procedures and expensive instruments, making it infeasible for point-of-care (POC) analysis especially in resourcelimited settings. In this work, a multicolorimetric ELISA biosensor integrated on a paper/polymer hybrid microfluidic device was developed for rapid visual detection of disease biomarkers at point of care, without using costly equipment. This multicolormetric ELISA platform was built on multiple distinct color variants resulted from the catalytic oxidation of 3,3',5,5'tetramethylbenzidine (TMB) and the etching of gold nanorods (AuNRs). The vivid color changes could be easily distinguished by the naked eye, and their red mean values allowed quantitative biomarker detection, without using any sophisticated instruments. When this multicolorimetric ELISA was integrated on a paper/polymer hybrid analytical device, it not only provided integrated processing and high portability but also enabled fast assays in about 50 min due to the unique advantages of paper/ polymer hybrid devices. The limit of detection of $9.1 \mathrm{ng} / \mu \mathrm{L}$ of the hepatitis $\mathrm{C}$ virus core antigen, a biomarker for hepatitis $\mathrm{C}$, was achieved using this multicolorimetric ELISA platform. This multicolor ELISA analytical device provides a new versatile, userfriendly, affordable, and portable immunosensing platform with high potential for on-site detections of various viruses, proteins, and biomarkers for low-resource settings such as at home, public venues, rural areas, and developing nations.
\end{abstract}

Keywords Multicolorimetric ELISA · Paper/polymer hybrid microfluidic device · Point-of-care detection · Quantitative biomarker detection $\cdot$ Infectious disease

\section{Introduction}

Hepatitis $\mathrm{C}$ is a liver disease caused by the hepatitis $\mathrm{C}$ virus (HCV), a hepatotropic flavivirus. The virus can cause both acute and chronic hepatitis, ranging in severity from a mild

Published in the topical collection Analytical Chemistry for Infectious Disease Detection and Prevention with guest editors Chaoyong Yang and XiuJun (James) Li.

Lei Ma and Yousef Abugalyon contributed equally to this work.

XiuJun Li

xli4@utep.edu

1 Deparment of Chemistry and Biochemistry, The University of Texas at El Paso, 500 West University Ave, El Paso, TX 79968, USA

2 Department of Chemistry and Biochemistry, Border Biomedical Research Center, Environmental Science and Engineering, University of Texas at El Paso, 500 West University Ave, El Paso, TX 79968, USA illness lasting a few weeks to a serious, lifelong illness. Chronic HCV infections often advance into cirrhosis or liver cancer. Due to its widespread, especially in underdeveloped and developing countries, each year about 399,000 HCVinfected people succumb to cirrhosis, hepatocellular carcinoma, and liver failure [1]. Therefore, screening of HCV infection is mandatory in many epidemiologic settings to begin appropriate treatment. There is an urgent need for rapid HCV detection so that the drugs can get administered to patients quickly, thus decreasing the chance of the patient getting other syndromes [2, 3]. Although advanced and sophisticated diagnostic technologies, including polymerase chain reaction (PCR) and enzyme-linked immunosorbent assay (ELISA), are extensively used in developed countries, they are not widely available in developing countries because of costly equipment, limited funds, and lack of skilled technicians. These techniques, along with other methods such as cell culture and western blotting, are normally laborious and timeconsuming [4-6]. Hence, rapid and sensitive point-of-care 
(POC) detection of HCV in low-resource settings (e.g., highpoverty regions) is still a challenge $[7,8]$.

ELISA is a widely used conventional technique that allows people to detect and quantify substances such as cells, viruses, and molecular antigens due to their specific binding to immobilized antibodies or antigens [4, 9-11]. However, conventional ELISA takes hours to complete because of the hourlong incubation (e.g., overnight) and blocking time. The consumption of large volumes of precious samples and reagents and the dependence on costly laboratory settings make ELISA not suitable for POC detection [6, 12]. Furthermore, the normally colorless property of ELISA reagents and monochromatic solutions with different intensities makes it hard to do the quantitative or semi-quantitative analysis without combining other detection technologies such as absorbance spectra, chromatography, radiolabeling, and sodium dodecyl sulphate-polyacrylamide gel electrophoresis (SDS-PAGE) $[10,11,13,14]$. This poses another problem of using expensive and bulky instruments, such as spectrophotometers and microplate readers [14-18], which significantly limits their application in POC detection in developing countries. Therefore, a portable, versatile, cost-effective, and instrumental-free ELISA method is highly desirable.

The multicolorimetric ELISA method can be a promising solution that can eliminate the use of these expensive and bulky instruments and allow it to be portable and cost-effective. Particularly, multicolorimetric sensing based on noble metal nanomaterial such as AuNRs [19] has attracted great interest owing to its advantages of visual color readout, simplicity, and robust enzymatic properties of nanomaterials, and those methods have been applied to detect various biomolecules and pathogens, such as pesticides, glucose, and Listeria monocytogenes [20-23]. For instance, a multicolorimetric sensor was developed for the detection of a toxic organophosphorus pesticide, omethoate, in a centrifuge tube based on the inhibition of the enzyme-induced metallization of AuNRs [20]. However, a microplate reader was required to achieve the quantitative analysis based on the wavelength shift. In recent studies, Lin et al. [21] presented a multicolor glucose sensor on a microplate, which allowed people to detect their glucose levels in serum based on different colors corresponding to different concentrations of glucose in sample solutions at the end of the assay. But an ultraviolet-visible spectrometer (UV-Vis) was still needed, which compromised its capability for point-of-care analysis. Liu et al. [22] reported another multicolorimetric aptasensor to determine the amount of Listeria monocytogenes in a centrifuge tube. However, the procedures to prepare samples and aptamer-conjugated magnetic nanoparticles were complex, and the assay required centrifugation and magnetic separation, which significantly prolonged the assay time and had stringent equipment requirements. However, these multicolorimetric assays were performed in a microplate or a centrifuge tube, which consumes more reagents and lacks integration and portability. Furthermore, these multicolorimetric assays are not ELISA; multicolorimetric ELISA is rarely reported. Although Li and co-workers reported a multicolor ELISA based on alkaline phosphatase-triggered growth of AuNRs, it still required overnight incubation and a microplate reader for quantitative analysis [24].

The recent microfluidic lab-on-a-chip technique provides a versatile platform for various bioanalyses particularly for POC analysis with the advantages of low reagent consumption, fast analysis, and high portability [25-33]. Paper-based microfluidic devices are low cost [2, 34, 35]; however, they have low performance in reagent control and handling, especially for ELISA that usually involves repeated washing steps. Because different chip substrates have different advantages and limitations, different paper/polymer hybrid microfluidic devices were developed to draw more benefits from two different substrates [36-41]. For instance, the novel introduction of paper in a paper/polymer hybrid microfluidic device significantly decreased the ELISA duration from approximately 30 to $1 \mathrm{~h}[13,14]$. In a paper/polymer hybrid microfluidic device, the paper provides a $3 \mathrm{D}$ porous substrate for rapid antibody immobilizations, ELISA conjugations, and many other reactions. Leveraging those advantages of paper/polymer hybrid microfluidic devices, the combination of a paper/polymer hybrid microfluidic chip with multicolor ELISA provides new opportunities for low-cost and rapid POC detection of $\mathrm{HCV}$ and other infectious diseases.

In this study, we developed a multicolorimetric ELISA biosensor on a paper/polymer hybrid analytical device for visual POC detection of HCV, based on multiple color variants through etching of AuNRs and the oxidation of 3,3',5,5'tetramethylbenzidine (TMB). As shown in Fig. 1, the catalytic oxidation of TMB via horseradish peroxidase (HRP) or other enzymes produced oxidized $\mathrm{TMB}^{2+}$, being accompanied by a distinct color change of the system [5]. Meanwhile, oxidized $\mathrm{TMB}^{2+}$ could etch AuNRs quickly, resulting in another color change. During the multicolorimetric ELISA in each microwell of the paper/hybrid chip, the presence of $\mathrm{HCV}$ was specifically recognized and captured by a monoclonal antibody and then immunocomplexed with the HRP conjugated secondary antibody. Different amounts of HCV produced different amounts of $\mathrm{TMB}^{2+}$, which modulated different degrees of AuNRs etching processes. Consequentially, the combination of the AuNRs etching and the TMB redox reaction resulted in various color changes, which could be easily discerned by the naked eye. The red mean values analyzed using ImageJ were introduced for the first time in the multicolorimetric ELISA for quantitative detection of $\mathrm{HCV}$, eliminating specialized analytical detectors such as spectrometers commonly used in other quantitative multicolorimetric assays. The limit of detection of $9.1 \mathrm{ng} / \mathrm{mL}$ of the HCV core antigen (HCVcAg) was successfully achieved without using 


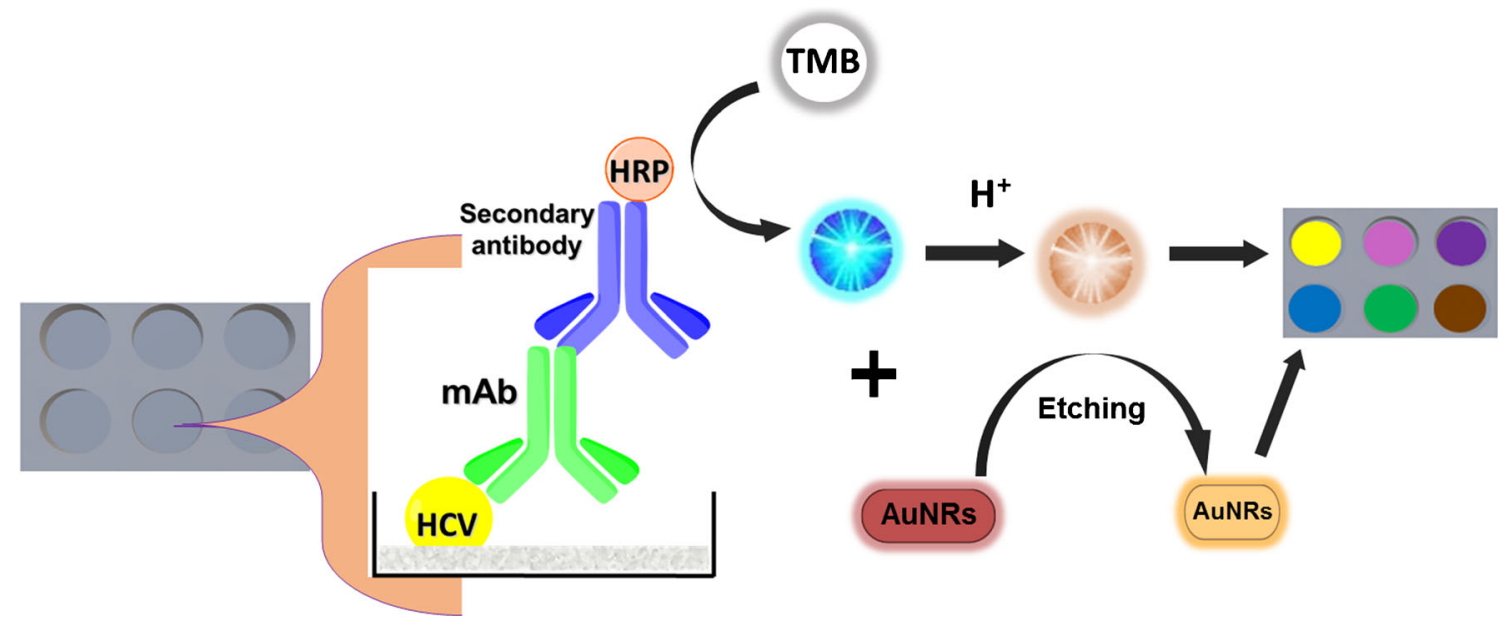

Fig. 1 Schematic showing the principle of the TMB/AuNRs-based multicolorimetric ELISA. The multicolorimetric ELISA is based on an indirect ELISA with HCV being the antigen and the HRP conjugated $\mathrm{IgG}$ as the secondary detection antibody

bulky and costly equipment. This on-chip multicolorimetric ELISA biosensor is rapid $(<50 \mathrm{~min})$ and portable, and allows rapid quantitative ELISA of infectious diseases at the point of care. This integrated multicolorimetric biosensor could be broadly applied to other target analytes with POC prospect, holding great potential for a wide range of applications.

\section{Experimental}

\section{Materials and reagents}

Silver nitrate $\left(\mathrm{AgNO}_{3}\right)$, ascorbic acid, 3, 3', 5, 5'tetramethylbenzidine (TMB), phosphate-buffered saline (PBS), poly(L)lysine, sodium hydroxide $(\mathrm{NaOH})$, dimethyl sulfoxide (DMSO), and human serum were purchased from m SigmaAldrich (Burlington, MA, USA). Sodium borohydride $\left(\mathrm{NaBH}_{4}\right)$, hydrochloric acid $(\mathrm{HCl})$, horseradish peroxidase (HRP), and glutaraldehyde were purchased from VWR International (Aurora, $\mathrm{CO}$, USA). The $\mathrm{HCV}$ core antigen (HCVcAg) and HCV monoclonal antibody were purchased from Abcam (Cambridge, MA, USA). Hydrogen tetrachloroaurate (III) hydrate, cetyl trimethyl ammonium bromide (CTAB), and IgG-HRP secondary antibody (rabbit) were purchased from Fisher Scientific International (Waltham, MA, USA). All aqueous solutions were prepared using ultrapure water purified by a Milli-Q system (Millipore, Bedford, MA, USA).

\section{Synthesis of the AuNRs}

The AuNRs were synthesized following the previously published seed-mediated method with a slight modification [42, 43]. Briefly, AuNRs were produced with the use of $25 \mathrm{~mL}$ of $\mathrm{HAuCl}_{4}(50 \mathrm{mM})$ mixed in $4.7 \mathrm{~mL}$ of CTAB $(0.1 \mathrm{M})$ in a $15-\mathrm{mL}$ centrifuge tube. After the addition of $\mathrm{AgNO}_{3}(10 \mathrm{mM})$ $100 \mathrm{~mL}, 30 \mathrm{~mL}$ of the $\mathrm{Au}$ seed solution was added into the
AuNRs growth solution in the centrifuge tube, and they were placed in a water bath at $30^{\circ} \mathrm{C}$ for a minimum of $2 \mathrm{~h}$ before use.

\section{Chip design and fabrication}

The three-layer paper/PMMA hybrid chip shown in Fig. $2 b$ was prepared following our previously published study [13]. Briefly, the top two PMMA plates were designed and modulated with Adobe Illustrator CS5 and laser ablated using a laser cutter machine (Epilog Laser, Golden, CO, USA). $6 \times$ 6 microwells $(6 \mathrm{~mm}$ in diameter) were laser ablated for the multicolorimetric ELISA for the higher throughput analysis. The top two layers were then assembled and sealed with a bottom plate through thermo-adhesion at $130^{\circ} \mathrm{C}$ by using a gravity convection oven (VWR International, Aurora, CO, USA). Meanwhile, the chromatography paper was cut into separate paper disks $(\Phi 6 \mathrm{~mm})$ by a laser cutter and placed into each microwell (3.97 $\mathrm{mm}$ in depth), forming a paper/ polymer hybrid analytical device, as shown in the crosssection view in Fig. 2a. An assembled hybrid chip filled with oxidized TMB (blue color) is displayed in Fig. 2c.

\section{Multicolorimetric ELISA procedures}

The TMB- $\mathrm{H}_{2} \mathrm{O}_{2}$ solution was firstly prepared, consisting of $0.5 \mathrm{mg}$ of TMB, $1 \mathrm{~mL}$ of DMSO, $9 \mathrm{~mL}$ of PBS (pH 7.4), and $2 \mathrm{~mL}$ of hydrogen peroxide $\left(\mathrm{H}_{2} \mathrm{O}_{2}\right)(30 \%)$. The HRP solutions at various concentrations from 0.0125 to $0.25 \mathrm{U} / \mathrm{mL}$ were prepared through multiple steps of dilutions with PBS. In each well of the chip, before the ELISA, the TMB- $\mathrm{H}_{2} \mathrm{O}_{2}$ solution and the HRP solution were mixed with a ratio of $15: 1$. After 5 min incubation, $0.05 \mathrm{M} \mathrm{HCl}$ was added to quench the reaction between TMB and $\mathrm{H}_{2} \mathrm{O}_{2}$. Ultimately, the AuNRs ( $\left.2.67 \mathrm{nM}\right)$ solution was added and became etched, resulting in different color changes from different concentrations of the oxidized $\mathrm{TMB}^{2+}$. The multicolorimetric ELISA was firstly tested on a microplate, and the colorimetric 
Fig. 2 a Cross-section view and $\mathbf{b}$ top view of the paper/PMMA hybrid chip for multicolor ELISA. c Photograph of an assembled device with oxidized TMB solutions in wells
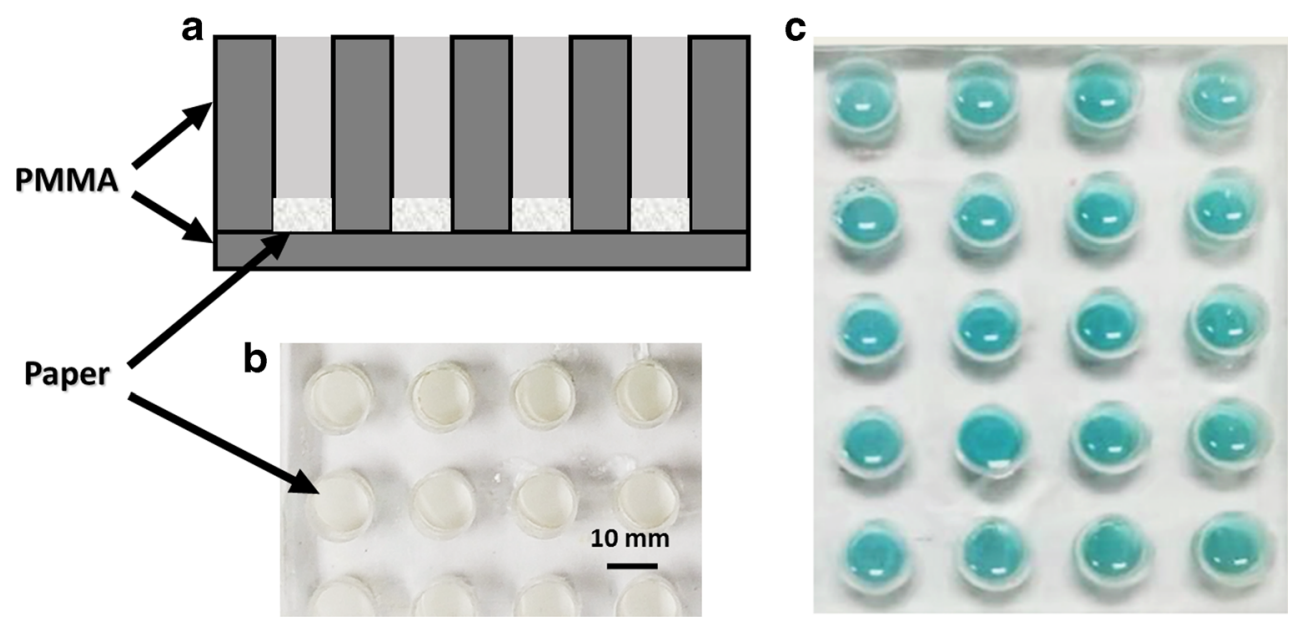

variations were characterized using UV-Vis spectra via a microplate reader (Molecular Devices, Sunnyvale, CA, USA) prior to on-chip detection. The final multicolorimetric detection results from on-chip ELISA were captured by Digital Single Lens Reflex Nikon D3500 (Nikon Corporation, Tokyo, Japan) or a smartphone camera [44], and analyzed by ImageJ $(n=5)$. See Supplementary Information (ESM) for more details in ImageJ analysis.

\section{Results and discussions}

\section{Condition optimization of the TMB- $\mathrm{H}_{2} \mathrm{O}_{2}$ colorimetric system}

To achieve optimal color changes from this multicolorimetric system, multiple conditions of the TMB- $\mathrm{H}_{2} \mathrm{O}_{2}$ were optimized, including TMB reaction time, $\mathrm{H}_{2} \mathrm{O}_{2}$ concentrations, and $\mathrm{HCl}$ concentrations.

The optimization of the TMB reaction time is a crucial aspect of the assay and was processed firstly, because the reaction time between TMB and HRP can significantly affect color changes from the TMB- $\mathrm{H}_{2} \mathrm{O}_{2}$ solution. We first tested the color changes of the TMB- $\mathrm{H}_{2} \mathrm{O}_{2}$ system at different reaction times in the absence of AuNRs. As shown from Fig. 3b, the 5-min reaction time allowed us to obtain more varieties of colors including from yellow, green to blue, while the 20-min reaction time only resulted in color in blue or colorless. The HRPcatalyzed TMB- $\mathrm{H}_{2} \mathrm{O}_{2}$ system is a commonly used ELISA, in which absorbance at $650 \mathrm{~nm}$ is usually used for the target quantitation and characterization of the reaction system [5]. Hence, the UV-Vis absorbance of these samples with different concentrations of HRP after the 5-min reaction time was measured and plotted in Fig. 3a, which also shows the absorbance of the TMB solution at $650 \mathrm{~nm}$ was located in the middle having greater dynamic change potential among these tested samples. Therefore, 5 min was chosen as the optimal TMB reaction time.
The concentration of $\mathrm{H}_{2} \mathrm{O}_{2}$ was optimized as well, because hydrogen peroxide played the major role of oxidizing TMB in the presence of HRP. We tested the color changes and UV-Vis absorbance of the TMB- $\mathrm{H}_{2} \mathrm{O}_{2}$ system in the presence of varying concentrations of $\mathrm{H}_{2} \mathrm{O}_{2}$. Similarly, we can find from Fig. $3 \mathrm{~d}$ that the $2.22 \%$ of $\mathrm{H}_{2} \mathrm{O}_{2}$ provided us more color variations, compared with other concentrations of $\mathrm{H}_{2} \mathrm{O}_{2}$. The absorbance in 450nm wavelength that implied the status of the TMB secondary oxidation (Fig. 3c) increased with the concentration increase of $\mathrm{H}_{2} \mathrm{O}_{2}$, and the absorbance from $2.22 \% \mathrm{H}_{2} \mathrm{O}_{2}$ was located in the middle, allowing a wide range of dynamics accordingly. Considering the color changes and the spectra, we chose $2.22 \% \mathrm{H}_{2} \mathrm{O}_{2}$ for the multicolorimetric assay.

The concentration effect of $\mathrm{HCl}$ was also investigated because of the HRP inactivation role of $\mathrm{HCl}$, and consequently halting the oxidation of TMB to the secondary state (yellow). Simultaneously, $\mathrm{HCl}$ acts as a secondary proton donor to TMB, inducing $\mathrm{TMB}^{+}$to be further oxidized into $\mathrm{TMB}^{2+}$. Therefore, the $\mathrm{HCl}$ concentration was optimized and the results are shown in Fig. $4 \mathrm{a}$ and b. The images from Fig. 4a exhibit more color variants from $0.05 \mathrm{M} \mathrm{HCl}$. Because the color changes are essentially affected by the red green blue (RGB) values in an image, we analyzed their RGB values by using ImageJ, and the red mean values are plotted in Fig. 4b (more RGB discussions in the following sections). The red mean values from $0.05 \mathrm{M} \mathrm{HCl}$ show three distinct dynamic ranges with more differences in adjacent HRP concentrations, which was consistent with results from Fig. $4 \mathrm{a}$ and provided more color variants for semi-quantitative analysis. Given all factors above, $0.05 \mathrm{M}$ $\mathrm{HCl}$ was adopted for the TMB- $\mathrm{H}_{2} \mathrm{O}_{2}$ colorimetric assay.

\section{Optimization of the concentrations of AuNRs}

After the condition optimization of the TMB- $\mathrm{H}_{2} \mathrm{O}_{2}$ colorimetric reaction, the concentrations of AuNRs, the other major color influencer in the multicolor TMB/AuNRs system, were optimized as well. As mentioned earlier and illustrated in Fig. 


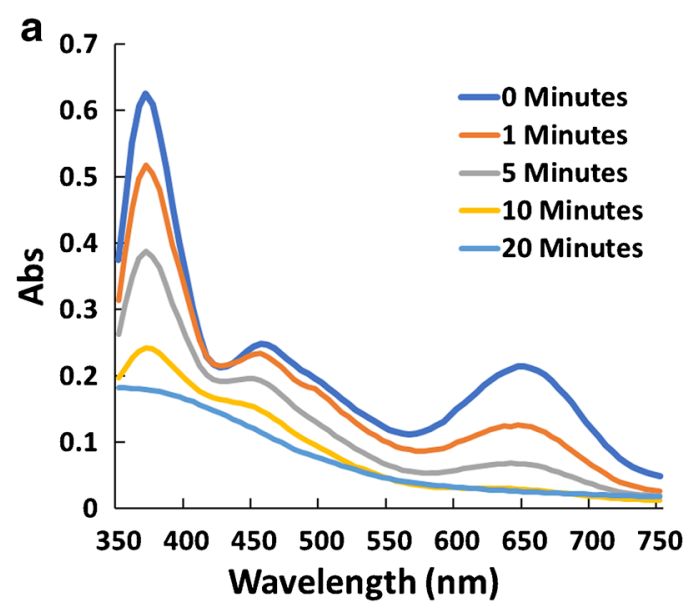

b

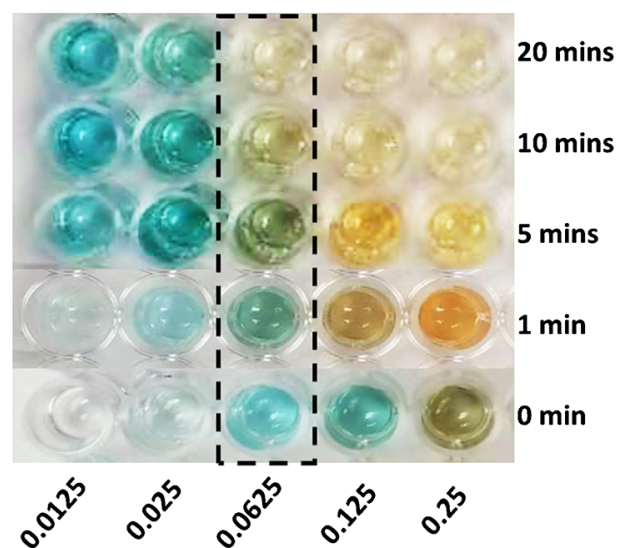

HRP concentration $\mathrm{U} / \mathrm{mL}$

Fig. 3 a Absorbance spectra and b scanned images of the TMB- $\mathrm{H}_{2} \mathrm{O}_{2}$ solutions at different reaction times in the presence of different concentrations of HRP. Samples with $0.0625 \mathrm{U} / \mathrm{mL}$ HRP from the dashed quadrilateral area in a microplate were scanned by UV-Vis absorbance and are shown in a. c Absorbance spectra and $\mathbf{d}$ scanned images of

1, the produced $\mathrm{TMB}^{2+}$ will react with AuNRs to produce TMB; meanwhile, AuNRs (reddish/purple) will be oxidized into $\mathrm{Au}(\mathrm{I})$ in yellow. Different levels of etching of AuNRs

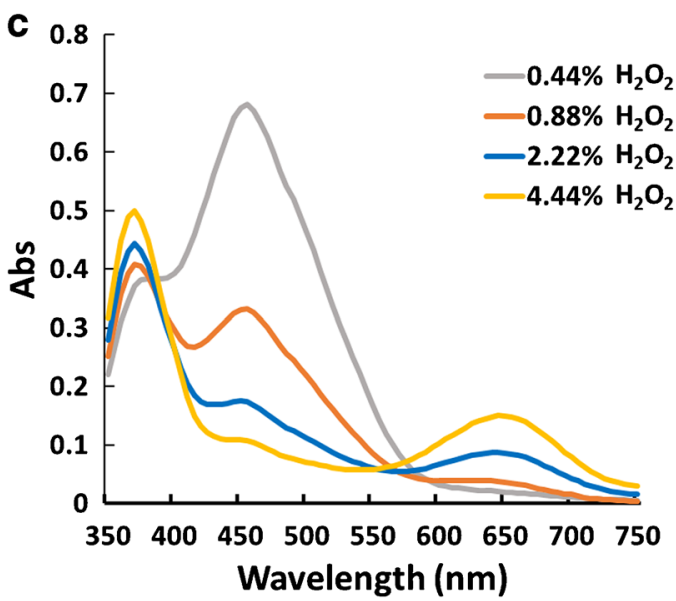

d

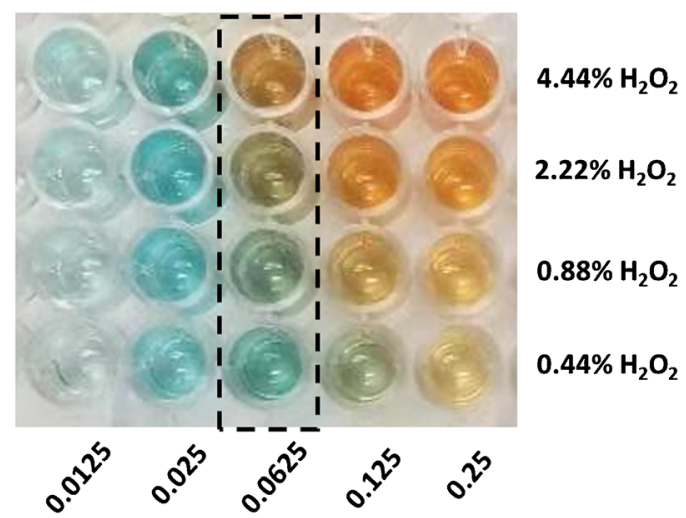

HRP concentration $\mathrm{U} / \mathrm{mL}$

the TMB- $\mathrm{H}_{2} \mathrm{O}_{2}$ solution at different concentrations of $\mathrm{H}_{2} \mathrm{O}_{2}$ in the presence of different concentrations of HRP. Samples with $0.0625 \mathrm{U} / \mathrm{mL}$ HRP from the dashed quadrilateral area were scanned by UV-Vis absorbance and are shown in $\mathbf{c}$

will result in various colors of the solution, i.e., the original concentration of AuNRs will affect the color of the solution collectively. Hence, we tested the color changes of the TMB/
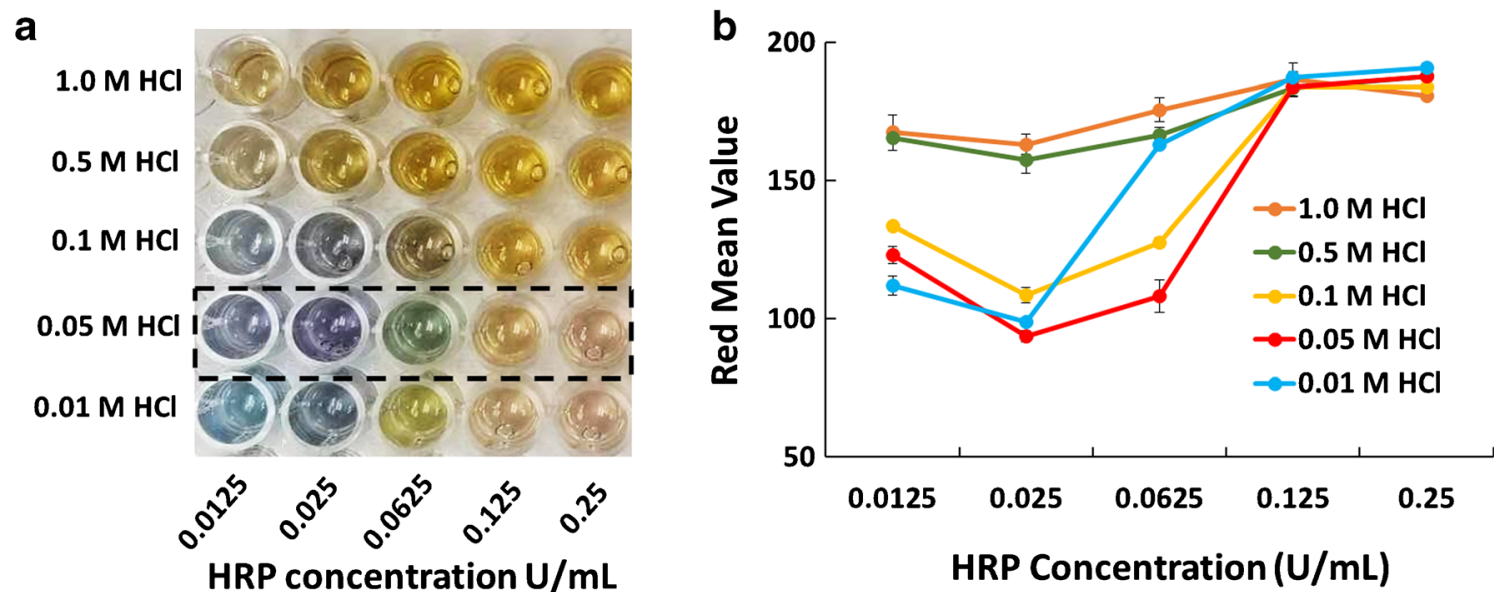

Fig. 4 a Partial scanned image and $\mathbf{b}$ graph of the red mean values of the TMB- $\mathrm{H}_{2} \mathrm{O}_{2}$ solutions in the presence of varying concentrations of $\mathrm{HCl}$. The red mean values of those samples in the dashed quadrilateral areas were plotted in $\mathbf{b}$. Error bars represent SDs of 5 different replicates 
AuNRs multicolorimetric system with varying concentrations of AuNRs in the presence of different concentrations of the secondary detection antibody conjugated with HRP (i.e., IgGHRP). It can be seen from Fig. 5a that a light blue color started to be perceived for all three concentrations of AuNRs from the lower concentrations of $0.00098 \mathrm{U} / \mathrm{mL}$ IgG-HRP, while higher concentrations of IgG-HRP caused darker blue, green, and light yellow. The clear color in the leftmost two microwells in each row indicates that those two HRP concentrations are not distinguishable by the naked eye (or below the limit of detection) under our TMB/AuNRs colorimetric conditions. Since the low concentration of $0.191 \mu \mathrm{g} / \mathrm{mL}$ AuNRs was capable of indicating similar color changes as other higher concentrations of AuNRs did in our TMB/AuNRs multicolorimetric system, we used $0.191 \mu \mathrm{g} / \mathrm{mL}$ AuNRs for the subsequent experiments. These different color variants in Fig. 5a also demonstrated the feasibility of our TMB/AuNRs multicolorimetric ELISA for semi-quantitative bioanalysis.

To achieve the quantitative analysis of the multicolorimetric ELISA without using specialized equipment, we studied the RGB values of these three different colors, light blue, green, and yellow. As listed in ESM Table S1, red mean values of light blue, dark green, and yellow are 80,0 , and 255 , respectively. In contrast, the green values of these three colors are 208, 192, and 224 , respectively. Among RGB values, red mean values give the best indicators to distinguish these three colors. Therefore, red mean values were chosen in this work to explore the quantitative multicolorimetric ELISA without using any costly and bulky equipment in the subsequent assays. The red mean values of TMB/AuNRs ELISA solutions in the top row of Fig. 5a were analyzed by using ImageJ and are shown in Fig. 5b. At the AuNRs concentration of $0.191 \mu \mathrm{g} / \mathrm{mL}$, the red mean values showed a linear relationship along with different concentrations of IgG-HRP (from $4.90 \times 10^{-3}$ to $4.90 \times 10^{-2} \mathrm{U} / \mathrm{mL}$ ), with the $R^{2}$ values of $0.99(P \leq 0.05)$, indicating the feasibility of using red mean values for quantitative analysis in this multicolorimetric ELISA. Hence, quantitative multicolorimetric ELISA can be achieved without using sophisticated equipment, a bottleneck problem in current quantitative multicolorimetric assays.

\section{Multicolorimetric ELISA biosensor on a paper/polymer hybrid device}

After condition optimization of TMB and AuNRs multicolorimetric system, we performed complete multicolorimetric ELISAs of different concentrations of the
Fig. 5 a Scanned image of the $\mathrm{TMB} /$ AuNRs solutions with different AuNRs concentrations, among which $0.191 \mu \mathrm{g} / \mathrm{mL}$ AuNRs allowed for sensitive detection of HRP in the presence of $2.22 \% \mathrm{H}_{2} \mathrm{O}_{2}$. b The correlation between the red mean values and IgG-HRP concentrations from $4.90 \times 10^{-3}$ to $4.90 \times 10^{-2} \mathrm{U} / \mathrm{mL}$ for different solutions at the AuNRs concentration of $0.191 \mu \mathrm{g} / \mathrm{mL}$. Error bars represent SDs of 5 different replicates
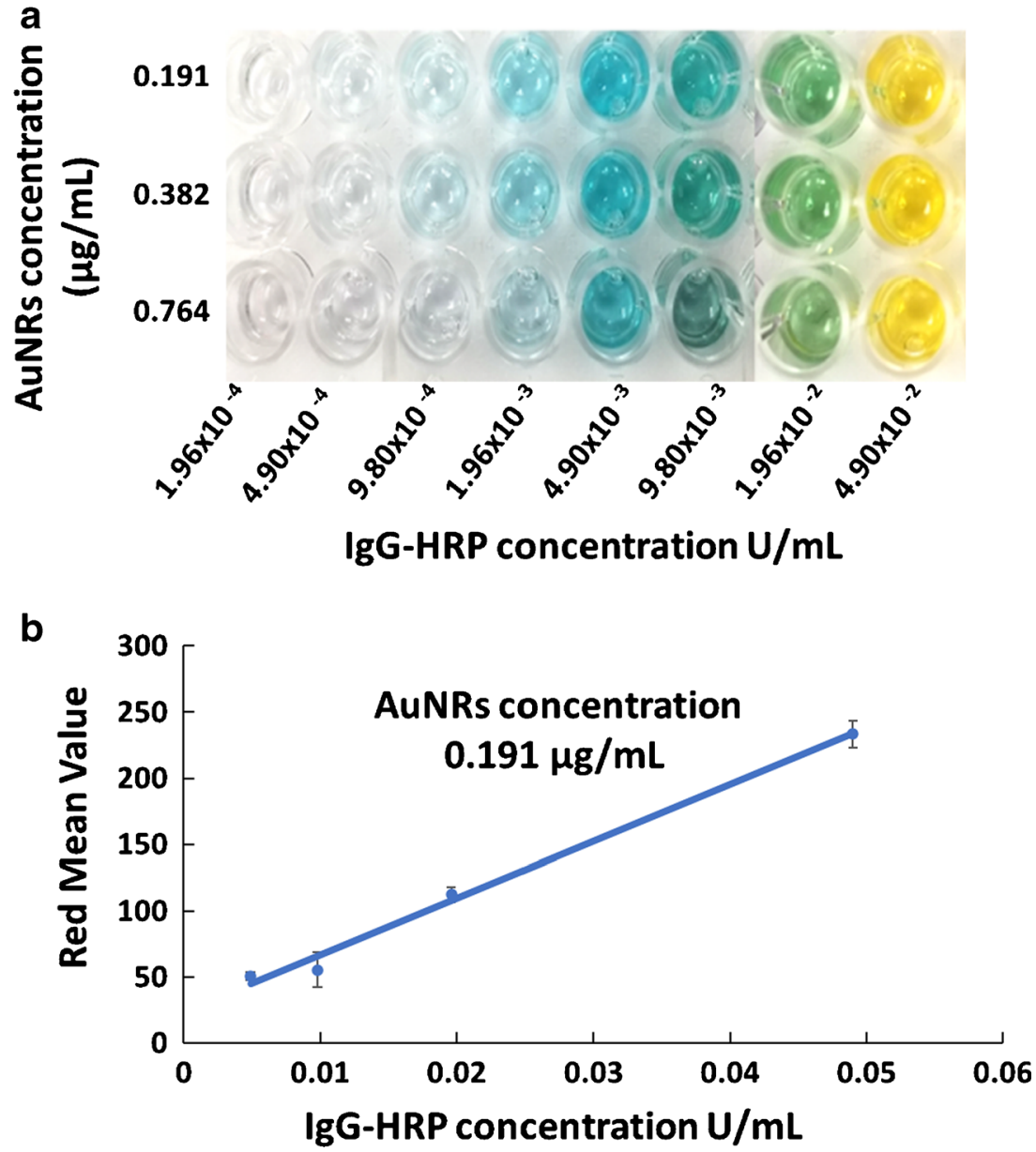
target $\mathrm{HCV}$ on the paper/polymer hybrid analytical device, following a protocol depicted in Fig. 1. Briefly, the HCV core antigen $(0-100 \mathrm{ng} / \mathrm{mL}$ in $10 \mu \mathrm{L}$ PBS pH 7.4 solution) was added to the microwells of the paper polymer hybrid chip. Then, 10 min of incubation was processed for the antigen immobilization on the chromatography paper in the microwells. This 3D porous paper substrate could fasten the antigen immobilization process, decreasing the incubation time from overnight to $10 \mathrm{~min}$, which is a unique benefit of our paper/ polymer hybrid microfluidic device [13]. Then, the paper surface was blocked by a blocking buffer (4\% BSA w/v in PBS) for $7 \mathrm{~min}$. After washing with PBS (10 mM, pH 7.4), HCV monoclonal antibody and anti-rabbit IgG-HRP $(20 \mu \mathrm{g} / \mathrm{mL})$ were introduced, incubated (10 $\mathrm{min})$, and washed, sequentially. Finally, the TMB- $\mathrm{H}_{2} \mathrm{O}_{2}$ solution $(75 \mu \mathrm{L})$ was added and incubated for $5 \mathrm{~min}$, followed with the addition of $0.05 \mathrm{M} \mathrm{HCl}$ $(2 \mu \mathrm{L})$ and $0.191 \mu \mathrm{g} / \mathrm{mL}$ AuNRs $(20 \mu \mathrm{L})$. After $30 \mathrm{~s}$ at room temperature in the paper/polymer hybrid chip, the color variations from different concentrations of the target were captured (Fig. 6a) and their red mean values were analyzed by using ImageJ (Fig. 6b). As shown in Fig. 6a, as the concentrations of the HCV core antigen increased, the color showed vivid color changes from dark green to light yellow, allowing for semi-quantitative HCV detection by the on-chip multicolorimetric ELISA biosensor with the naked eye.

a
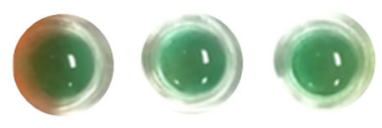

$\mathbf{0}$

10

30



50

HCVcAg concentrations $(\mathrm{ng} / \mathrm{mL})$

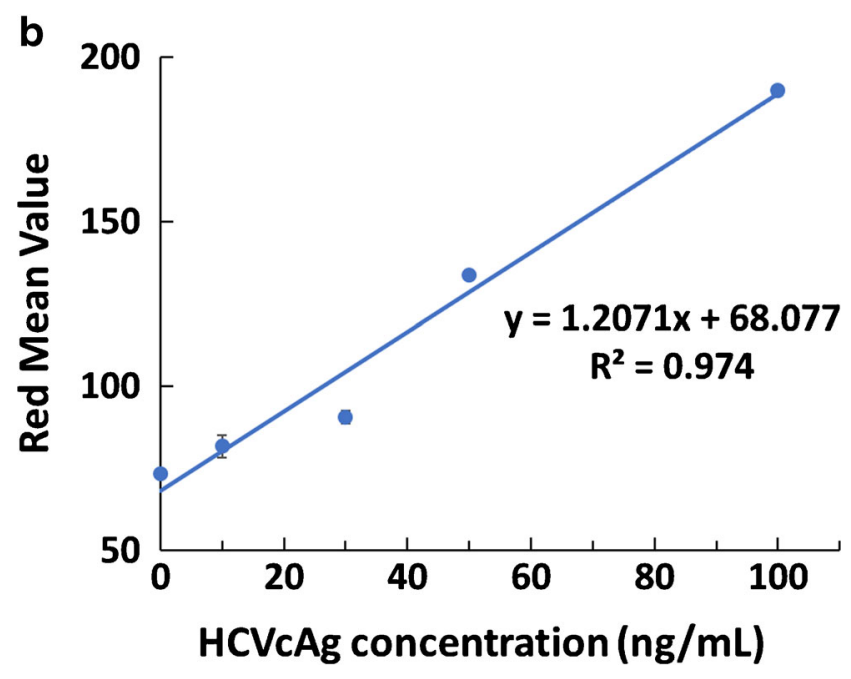

Fig. 6 Quantitative detection of HCV by the multicolorimetric ELISA biosensor on the paper/polymer hybrid device without using specialized equipment. a Partial scanned images of the on-chip test of various solutions with varying concentrations of $\mathrm{HCVcAg}$. b HCVcAg calibration curve between the red mean values versus HCVcAg concentrations from 0 to $100 \mathrm{ng} / \mathrm{mL}(n=5)$
Table 1 Determination of HCVcAg spiked in human serum samples $(n=4)$

\begin{tabular}{llll}
\hline Sample & $\begin{array}{l}\text { Added HCVcAg } \\
(\mathrm{ng} / \mathrm{mL})\end{array}$ & $\begin{array}{l}\text { Detected } \\
\text { HCVcAg }(\mathrm{ng} / \mathrm{mL})\end{array}$ & Recovery (\%) \\
\hline $\begin{array}{c}\text { Human serum } \\
\text { samples }\end{array}$ & 0 & N.D. & - \\
& 10 & 10.45 & $104.53 \pm 1.54$ \\
& 50 & 50.78 & $101.55 \pm 2.55$ \\
& 100 & 98.02 & $98.02 \pm 1.47$ \\
\hline
\end{tabular}

N.D., not detectable

To achieve quantitative multicolorimetric ELISA, as discussed earlier, the captured images were analyzed by ImageJ and their different red mean values were plotted as the calibration curve versus the concentration of $\mathrm{HCVcAg}$. As displayed in Fig. 6b, the red mean values increased with the increase of the $\mathrm{HCVcAg}$ concentrations. A linear relationship was obtained between the red mean values and the $\mathrm{HCVcAg}$ concentration in the range from 10.0 to $100.0 \mathrm{ng} /$ $\mathrm{mL}$, with the $R$-squared value of 0.974 . The limit of detection (LOD) of this on-chip multicolorimetric ELISA was calculated to be as low as $9.1 \mathrm{ng} / \mathrm{mL}$ based on 3-fold standard deviations above the blank signal. Although this LOD is slightly higher than that of a conventional ELISA using a microplate reader (LOD, $1.0 \mathrm{ng} / \mathrm{mL}$ ) [45], this multicolorimetric ELISA not only provides semi-quantitative results in the form of different vivid colors to the naked eye but also allows quantitative disease detection without using bulky and costly instruments. All these features make the on-chip multicolorimetric ELISA biosensor particularly suitable for POC detection and other bioanalyses in resource-limited settings such as rural areas and developing nations.

To validate our method, three different concentrations (10, 50 , and $100 \mathrm{ng} / \mathrm{mL}$ ) of $\mathrm{HCVcAg}$ spiked in whole human serum were tested using our on-chip multicolorimetric ELISA biosensor and the results are listed in Table 1. The human serum samples without spiked HCVcAg spiked were not detectable by our method. Other spiked samples at concentrations of 10,50 , and $100 \mathrm{ng} / \mathrm{mL}$ of $\mathrm{HCVcAg}$ showed recovery percentages of $104.53 \%, 101.55 \%$, and $98.02 \%$, respectively. These results are within the acceptable range for the validation of analytical methods, which not only has validated the accuracy of our method but also indicates the robustness of our method in testing complex human samples.

\section{Conclusions}

A TMB/AuNRs-based multicolorimetric ELISA biosensor on a paper/polymer hybrid microfluidic device has been developed for rapid semi-quantitative and quantitative POC 
detection of $\mathrm{HCV}$ in low-resource settings, without using any bulky and costly instruments. The vivid multiple color variants resulted from the oxidation of TMB and the etching of AuNRs enabled visual semi-quantitative detection by the naked eye, while analyzed RGB values were successfully applied for quantitative detection of disease biomarkers, without the aid of specialized analytical detectors. Additionally, the ingenious introduction of paper in this hybrid multicolorimetric ELISA device greatly shortened the assay time from hours to about $50 \mathrm{~min}$. After condition optimization, the LOD of $9.1 \mathrm{ng} / \mu \mathrm{L}$ of HCVcAg, an HCV biomarker, was achieved using this multicolorimetric ELISA platform.

This multicolorimetric ELISA biosensor on a paper/ polymer hybridanalytical device is portable and low cost, and allows fast semi-quantitative and quantitative analysis without using costly specialized equipment. All these significant features make it highly desirable for POC detection and other bioanalyses, particularly for rural areas and developing nations. Furthermore, this multicolorimetric ELISA platform has great potential for POC detection of a variety of other infectious diseases from hepatitis B and tuberculosis (TB) to recent COVID-19 [13, 46, 47].

Supplementary Information The online version contains supplementary material available at https://doi.org/10.1007/s00216-021-03359-8.

Acknowledgements We would like to acknowledge the financial support from the National Institute of Allergy and Infectious Disease of the NIH (R21AI107415), the U.S. NSF (IIP2052347, IIP1953841, and DMR1827745), DOT (CARTEEH), the University of Texas at El Paso (UTEP) for the IDR Program, the Philadelphia Foundation, and the Medical Center of the Americas Foundation. We are also grateful for the financial support to our prior research from the National Institute of General Medical Sciences of the NIH (SC2GM105584), the NIH RCMI Pilot grant, the NIH BUILDing Scholar Summer Sabbatical Award, NSF, University of Texas (UT) System for the STARS award, and the University of Texas at El Paso (UTEP) for the Multidisciplinary Research Award Program (MRAP) and URI Program.

\section{Declarations}

Competing interests The authors declare no competing interests.

\section{References}

1. Jefferies M, Rauff B, Rashid H, Lam T, Rafiq S. Update on global epidemiology of viral hepatitis and preventive strategies. World journal of clinical cases. 2018;6(13):589.

2. Prasad KS, Abugalyon Y, Li C, Xu F, Li X. A new method to amplify colorimetric signals of paper-based nanobiosensors for simple and sensitive pancreatic cancer biomarker detection. Analyst. 2020;145(15):5113-7.

3. Dou M, Sanchez J, Tavakoli H, Gonzalez JE, Sun J, Dien Bard J, et al. A low-cost microfluidic platform for rapid and instrument-free detection of whooping cough. Anal Chim Acta. 2019;1065:71-8.

4. Sanjay ST, Li M, Zhou W, Li X, Li X. A reusable PMMA/paper hybrid plug-and-play microfluidic device for an ultrasensitive immunoassay with a wide dynamic range. Microsystems \& Nanoengineering. 2020;6(1):1-11.

5. Fu G, Sanjay ST, Zhou W, Brekken RA, Kirken RA, Li X. Exploration of nanoparticle-mediated photothermal effect of TMB-H2O2 colorimetric system and its application in a visual quantitative photothermal immunoassay. Anal Chem. 2018;90(9): 5930-7.

6. Fu G, Sanjay ST, Li X. Cost-effective and sensitive colorimetric immunosensing using an iron oxide-to-Prussian blue nanoparticle conversion strategy. Analyst. 2016;141(12):3883-9.

7. Albertoni G, Girao M, Schor N. Mini review: current molecular methods for the detection and quantification of hepatitis B virus, hepatitis $\mathrm{C}$ virus, and human immunodeficiency virus type 1 . Int $\mathrm{J}$ Infect Dis. 2014;25:145-9.

8. Fu G, Sanjay ST, Dou M, Li X. Nanoparticle-mediated photothermal effect enables a new method for quantitative biochemical analysis using a thermometer. Nanoscale. 2016;8(10): 5422-7.

9. Fu G, Li X, Wang W, Hou R. Multiplexed tri-mode visual outputs of immunoassay signals on a clip-magazine-assembled photothermal biosensing disk. Biosens Bioelectron. 2020;170: 112646.

10. Ma L, Tang B, Yang W, Liu Y, Zhao Y, Li M. Integration of a biochip technique with technetium-99m labeling provides zeptomolar sensitivity in liver cancer biomarker detection. Anal Methods. 2015;7(4):1622-6.

11. Tang B, Wang J, Hutchison JA, Ma L, Zhang N, Guo H, et al. Ultrasensitive, multiplex Raman frequency shift immunoassay of liver cancer biomarkers in physiological media. ACS Nano. 2016;10(1):871-9.

12. Kai J, Puntambekar A, Santiago N, Lee SH, Sehy DW, Moore V, et al. A novel microfluidic microplate as the next generation assay platform for enzyme linked immunoassays (ELISA). Lab Chip. 2012;12(21):4257-62.

13. Sanjay ST, Dou M, Sun J, Li X. A paper/polymer hybrid microfluidic microplate for rapid quantitative detection of multiple disease biomarkers. Sci Rep. 2016;6(1):30474.

14. Sanjay ST, Li M, Zhou W, Li X, Li X. A reusable PMMA/paper hybrid plug-and-play microfluidic device for an ultrasensitive immunoassay with a wide dynamic range. Microsystems \& Nanoengineering. 2020;6(1):28.

15. Patel J, Sharma P. Design of a novel rapid immunoassay for simultaneous detection of hepatitis $\mathrm{C}$ virus core antigen and antibodies. Arch Virol. 2020;165(3):627-41.

16. Hassanin TM, Abdelraheem EM, Abdelhameed S, Abdelrazik M, Fouad YM. Detection of hepatitis $\mathrm{C}$ virus core antigen as an alternative method for diagnosis of hepatitis $\mathrm{C}$ virus infection in blood donors negative for hepatitis $\mathrm{C}$ virus antibody. European Journal of Gastroenterology \& Hepatology. 9000; Publish Ahead of Print.

17. Kim DD, Hutton DW, Raouf AA, Salama M, Hablas A, Seifeldin IA, et al. Cost-effectiveness model for hepatitis $\mathrm{C}$ screening and treatment: implications for Egypt and other countries with high prevalence. Glob Public Health. 2015;10(3):296-317.

18. Datta S, Goel N, Wattal C. Utility of routine real time quantitative PCR monitoring of $\mathrm{HCV}$ infection in haemodialysis patients. Indian J Med Microbiol. 2015;33(Suppl):106-11.

19. Mohamad A, Teo H, Keasberry NA, Ahmed MU. Recent developments in colorimetric immunoassays using nanozymes and plasmonic nanoparticles. Crit Rev Biotechnol. 2019;39(1):50-66.

20. Zhang Q, Yu Y, Yun X, Luo B, Jiang H, Chen C, et al. Multicolor colorimetric sensor for detection of omethoate based on the inhibition of the enzyme-induced metallization of gold nanorods. ACS Applied Nano Materials. 2020;3(6):5212-9.

21. Lin Y, Zhao M, Guo Y, Ma X, Luo F, Guo L, et al. Multicolor colormetric biosensor for the determination of glucose based on the etching of gold nanorods. Sci Rep. 2016;6(1):37879. 
22. Liu Y, Wang J, Zhao C, Guo X, Song X, Zhao W, et al. A multicolorimetric assay for rapid detection of Listeria monocytogenes based on the etching of gold nanorods. Anal Chim Acta. 2019;1048:154-60.

23. Si Z, Li Y, Han S, Liu Y, Hu P, Lu S, et al. Gold nanorods-based multicolor immunosensor for visual detection of enterovirus 71 infection. Microchim Acta. 2020;187(10):1-8.

24. Li Y, Ma X, Xu Z, Liu M, Lin Z, Qiu B, et al. Multicolor ELISA based on alkaline phosphatase-triggered growth of $\mathrm{Au}$ nanorods. Analyst. 2016;141(10):2970-6.

25. Sanjay ST, Fu G, Dou M, Xu F, Liu R, Qi H, et al. Biomarker detection for disease diagnosis using cost-effective microfluidic platforms. Analyst. 2015;140(21):7062-81.

26. Tavakoli H, Zhou W, Ma L, Perez S, Ibarra A, Xu F, et al. Recent advances in microfluidic platforms for single-cell analysis in cancer biology, diagnosis and therapy. TrAC Trends Anal Chem. 2019;117:13-26.

27. Sanjay ST, Zhou W, Dou M, Tavakoli H, Ma L, Xu F, et al. Recent advances of controlled drug delivery using microfluidic platforms. Advanced Drug Delivery Reviews. 2018;128:3-28 (*Five-Year impact factor 17.3).

28. Lv M, Zhou W, Tavakoli H, Bautista C, Xia J, Wang Z, et al. Aptamer-functionalized metal-organic frameworks (MOFs) for biosensing. Biosens Bioelectron. 2021;176:112947.

29. Li XJ, Zhou Y. Microfluidic devices for biomedical applications: Woodhead Publishing; 2013.

30. Li XJ, Valadez AV, Zuo P, Nie Z. Microfluidic 3D cell culture: potential application for tissue-based bioassays. Bioanalysis. 2012;4(12):1509-25.

31. Zhang J, Wei X, Zeng R, Xu F, Li X. Stem cell culture and differentiation in microfluidic devices toward organ-on-a-chip. Future Science OA. 2017;3(2):FSO187.

32. Dou M, Macias N, Shen F, Dien Bard J, Domínguez DC, Li X. Rapid and accurate diagnosis of the respiratory disease pertussis on a point-of-care biochip. EClinicalMedicine. 2019;8:72-7.

33. Wei X, Zhou W, Sanjay ST, Zhang J, Jin Q, Xu F, et al. Multiplexed instrument-free bar-chart SpinChip integrated with nanoparticle-mediated magnetic aptasensors for visual quantitative detection of multiple pathogens. Anal Chem. 2018;90(16):988896.

34. Prasad KS, Cao X, Gao N, Jin Q, Sanjay ST, Henao-Pabon G, et al. A low-cost nanomaterial-based electrochemical immunosensor on paper for high-sensitivity early detection of pancreatic cancer. Sensors and Actuators B: Chemical. 2020;305:127516.
35. Zhou W, Feng M, Valadez A, Li X. One-step surface modification to graft DNA codes on paper: the method, mechanism, and its application. Anal Chem. 2020;92(10):7045-53.

36. Zuo P, Li X, Dominguez DC, Ye B-C. A PDMS/paper/glass hybrid microfluidic biochip integrated with aptamer-functionalized graphene oxide nano-biosensors for one-step multiplexed pathogen detection. Lab Chip. 2013;13(19):3921-8.

37. Li X, Dou M, Dominguez DC, inventors Methods and compositions for paper-based and hybrid microfluidic devices integrated with nucleic acid amplification for disease diagnosis. USA patent 10875024. 2020.

38. Dou M, Sanjay ST, Benhabib M, Xu F, Li X. Low-cost bioanalysis on paper-based and its hybrid microfluidic platforms. Talanta. 2015;145:43-54.

39. Tavakoli H, Zhou W, Ma L, Guo Q, Li X. Paper and paper hybrid microfluidic devices for point-of-care detection of infectious diseases. Nanotechnology and Microfluidics. 2020:177-209.

40. Dou M, Dominguez DC, Li X, Sanchez J, Scott G. A versatile PDMS/paper hybrid microfluidic platform for sensitive infectious disease diagnosis. Anal Chem. 2014;86(15):7978-86.

41. Dou M, Sanjay ST, Dominguez DC, Zhan S, Li X. A paper/ polymer hybrid CD-like microfluidic SpinChip integrated with DNA-functionalized graphene oxide nanosensors for multiplex qLAMP detection. Chem Comm. 2017;53(79):10886-9.

42. Wang J, Li M, Tang B, Xie P, Ma L, Hu Z, et al. Assembling single gold nanorods into large-scale highly aligned nanoarrays via vacuum-enhanced capillarity. Nanoscale Res Lett. 2014;9(1):1-5.

43. Scarabelli L, Sánchez-Iglesias A, Pérez-Juste J, Liz-Marzán LM. A "tips and tricks" practical guide to the synthesis of gold nanorods. The Journal of Physical Chemistry Letters. 2015;6(21):4270-9.

44. Xu X, Wang X, Hu J, Gong Y, Wang L, Zhou W, et al. A smartphone-based on-site nucleic acid testing platform at pointof-care settings. ELECTROPHORESIS. 2019;40(6):914-21.

45. QuickTiter HCV Core Antigen ELISA Kit. Cell Biolabs, Inc; 2021.

46. Zhou W, Sun J, Li X. Low-cost quantitative photothermal genetic detection of pathogens on a paper hybrid device using a thermometer. Anal Chem. 2020;92(21):14830-7.

47. Zhou W, Hu K, Kwee S, Tang L, Wang Z, Xia J, et al. Gold nanoparticle aggregation-induced quantitative photothermal biosensing using a thermometer: a simple and universal biosensing platform. Anal Chem. 2020;92(3):2739-47.

Publisher's note Springer Nature remains neutral with regard to jurisdictional claims in published maps and institutional affiliations. 